\title{
A Stackelberg-Game-Based Power Control Algorithm for Wireless Mesh Networks
}

\author{
Yanbing Liu, Tao Wu, Jun Huang, and Shousheng Jia \\ Chongqing Engineering Laboratory of Internet and Information Security, Chongqing University of Posts and \\ Telecommunications (CQUPT), Chongqing 400065, China
}

Correspondence should be addressed to Yanbing Liu; liuyb@cqupt.edu.cn

Received 25 October 2012; Accepted 4 December 2012

Academic Editor: Chuandong Li

Copyright (C) 2013 Yanbing Liu et al. This is an open access article distributed under the Creative Commons Attribution License, which permits unrestricted use, distribution, and reproduction in any medium, provided the original work is properly cited.

\begin{abstract}
Wireless mesh networks (WMNs) are a promising networking paradigm for next generation wireless networking system. Power control plays a vital role in WMNs and is realized to be a crucial step toward large-scale WMNs deployment. In this paper, we address the problem of how to allocate the power for both optimizing quality of service (QoS) and saving the power consumption in WMNs based on the game theory. We first formulate the problem as a noncooperative game, in which the QoS attributes and the power of each node are defined as a utility function, and all the nodes attempt to maximize their own utility. In such game, we correlate all the interfering nodes to be an interfering object and the receiving node to be the interfering object's virtual destination node. We then present an equilibrium solution for the noncooperative game using Stackelberg model, and we propose an iterative, distributed power control algorithm for WMNs. Also, we conduct numeric experiments to evaluate the system performance, our results show that the proposed algorithm can balance nodes to share the limited network resources and maximize total utility, and thus it is efficient and effective for solving the power control problem in WMNs.
\end{abstract}

\section{Introduction}

Wireless mesh networks (WMNs) are a key networking paradigm for next generation wireless networking system. The openness feature of WMNs makes the transmission power level affect the performance of wireless network significantly. In wireless networks, higher signal-to-interference plus noise ratio (SINR) value yields lower BER and higher data rate, and every node desires to have the lowest possible transmission power coupled with the highest SINR. Power control technology that deals with the selection of proper transmission power is closely related to quality of service (QoS) optimization and power saving. Therefore, power control plays a vital role in WMNs and is expected to be a crucial technique for large-scale WMNs deployment.

Power control scheme, which determines transmission range or interference range, is critical to the performance of the WMNs. However, the existing power control schemes that largely rely on the local information of sending node, which cannot accurately reflect the link quality of transmission path, and the transmissions with the power control schemes cannot share network resources reasonably. On the other hand, game theory, due to its mathematical capability in analyzing the interaction among independent decision-makers [1], has drawn much attention in the design of self-organizing and adaptive wireless networks. Game theory is a computing model by means of psychology, and it assumes that people are selfish, which is different from machine learning that try to discover the laws through the sample data. Game theory provides a powerful tool to study power allocation problems in competitive wireless networks. As far as we know, game theory has been actively applied for distributed resource allocation of different goals in adhoc and WMNs. Our research work is to devise a distributed, efficient power control scheme with low complexity for WMNs. In this paper, we propose a noncooperative power control algorithm for WMNs' QoS optimization and power saving using the Stackelberg game model. Specifically, the contributions made in this work are threefold.

(i) We design a multiobjective utility function to represent users' preference between QoS achieving and power saving, and we prove the existence and uniqueness of the theoretical optimal solution. 
(ii) Based on the Stackelberg game model, we formulate this power-allocation problem as a noncooperative game, and we solve the power control game to get subgame perfect Nash equilibrium (SPNE) in detail.

(iii) According to SPNE, we propose a distributed power control algorithm for WMNs. The algorithm can advantageously improve the network performance by balancing all nodes in the interference range to share the limited network resource.

Through numeric experiments, the proposed algorithm is shown to be efficient and effective in balancing nodes to share the limited network resources and maximizing total utility. We thus believe that it is applicable to various wireless networking systems.

The remainder of this paper is organized as follows. In Section 2, we present related works and the contributions of the work. Section 3 explicates the selfish character of WMNs nodes and designs a utility function for power control. Section 4 gives the system model and problem definition. Section 5 formulates the power control problem as the Stackelberg game model and conducts Nash equilibrium (NE) point. Section 6 presents the proposed power control algorithm. The numeric experiment is included in Section 7. Finally, we conclude the paper in Section 8.

\section{Related Work}

Recently a large amount of researches progress has been made in power allocation for wireless networks. Authors in [2] designed a power-controlled MAC protocol that enables nodes to adjust their transmission powers while allowing for some interference margin at the receiver. Simulation results demonstrated that significant throughput and energy gains are obtainable with MAC protocol. Nonetheless, it is difficult to implement synchronization between nodes. The MAC protocol fails to handle the interference problem as well. Literature [3] presented several distributed power control algorithms. These algorithms take the node sensitivities to current interference levels into account. In [4], the authors considered the problem of topology control in hybrid WMNs. An algorithm was developed to calculate the optimal transmission power so that network connectivity is maintained, node transmission power is reduced to cover only the nearest neighbors, and network lifetime is extended. However, the above referred research studies share the same features, that is, assuming the devices cooperate with the purpose of system performance optimization. While for the wireless nodes, they do not basically belong to a single authority and may not want to achieve consensus and pursue a common goal. Therefore, the nodes are usually too selfish like the players who attempt to maximize their own performance without considering the other players' objective. Therefore, the assumption might not hold.

Currently applying game theory in power allocation for competitive wireless networks has also become an active research topic. As far as we know, game theory has been actively applied for distributed resource allocation of different goals in ad hoc and WMNs. Tan and coauthors in [5] modeled the channel assignment and power control problem as a noncooperative game, in which all wireless users jointly pick an optimal channel and power level to minimize a joint cost function. However, the proposed algorithm is theoretic, and it cannot be implemented in practice conveniently. In [6], the authors proposed a payment-based power control scheme using game theory where each user announces a set of price coefficients that reflect different compensations paid by other users for the interference they produce. The proposed approach converges to Nash equilibrium where at this point it is able to provide a fairer throughput share among users. In [7], the authors focused on how to control the transmission power of the access points' pilot signals using game theory to manage interference. They considered a noncooperative power control game and computed the transmission power level of each access point as Nash equilibrium of the game. On the other hand, they considered a cooperative power control game and presented a punishment strategy enforced by the game regulator to punish selfish access points. However, the assumption of the game regulator is not feasible for WMNs. Since most of the terminals in a wireless network are battery powered, such WMNs clients and sensor nodes, energy efficiency is crucial to prolonging the life of the terminals. In addition to the PC game, studies pertinent to the energy efficiency game can be found in [8-10].

Through the aforementioned studies concentrated on QoS optimization (throughput optimization) and energy efficiency, little research has been reported to balance both the QoS optimization (throughput optimization) and energy efficiency under the game theoretic framework. In this paper, we address the problem of power allocation for both QoS optimization and power saving in wireless mesh networks. Our work differs from above researches in the following three ways. First, we address the problem in multihop WMNs instead of single-hop WMNs. Second, we present a joint objective function to balance the QoS optimization (throughput optimization) and energy efficiency. Third, we combine the QoS optimization and power saving into a single game termed noncooperative game (NQPG), and the detailed description is given in Section 5.

\section{Utility Function for Power Control}

In WMNs, each autonomous node attempts to maximize its own interest and tends to be "selfish"; therefore, there exists a game between these nodes. While in the power control scenario, a user tends to adjust its transmission power in response to other users' actions, which leads to a sequence of power vectors that converge to a point where no user has incentive to increase its individual power, and this point is called NE. Unfortunately, such an NE point does not necessarily exist. In general, we have the following sufficient conditions.

Definition 1. An NE exists in a noncooperative power control game $G=\left[N,\left\{P_{i}\right\},\left\{U_{i}(\cdot)\right\}\right]$ if, for all $i=1, \ldots, n$ :

(i) the strategy profile $P_{i}$ is a nonempty, convex, and compact subset of some Euclidean space $\mathbb{R}$,

(ii) $U_{i}(\cdot)$ is continuous and quasi concave in $P_{i}$, 
where $N=\{1,2, \ldots, n\}$ is the index set of the currently active nodes, $P_{i}$ represents the strategy set, and $U_{i}(\cdot)$ is the utility function for user $i$. Each node selects a power level $p_{i}$ from the convex set $P_{i}$ such that $p_{i} \in P_{i}$. Let the power vector $p=$ $\left(p_{1}, p_{2}, \ldots, p_{n}\right)$ denote the outcome of the game in terms of the selected power levels of all the users. The resulting utility level for the $i$ th user is $U\left(p_{i}, P_{-i}\right)$. The strategy space of all the users excluding the $i$ th user is denoted by $P_{-i}$.

In WMNs with shared transmission medium, the signal of one node may interfere with that of other nodes. Typically, a selfish user without any energy constraint would tend to achieve a high SINR at the receiving node irrespective of the fact that it may cause excessive interference to other users [5]. Eventually all nodes will transmit at their maximum power level because power is strictly increasing with the SINR. Considering the overall performance of the network, the desired utility function should take the SINR and power into account, so we specify a utility function for each node to adjust transmission power, which consists of three terms, and we will introduce them individually. To address the power control problem, we adopt a function to measure the throughput [11]:

$$
A_{1}\left(p_{i}, P_{-i}\right)=W \cdot \log _{2}\left(1+\frac{\gamma_{i}}{\Omega}\right),
$$

where $p_{i}$ denotes the strategy chosen by source node $i, P_{-i}$ denotes the strategies of all other nodes in interference range, $W$ is the link bandwidth, $\Omega$ is the channel gap, $\Omega \geq 1$, and $\gamma_{i}$ represents the SINR of node $i$ [5], and

$$
\gamma_{i}=\frac{p_{i} \cdot G_{i, j} / d_{i, j}^{\alpha}}{\sum_{k \in S_{-i}, k=1, k \neq i} p_{k} \cdot G_{k, j} / d_{k, j}^{\alpha}+N_{0}},
$$

where $d_{i, j}$ is the distance from sending node $i$ to receiving node $j, p_{i}$ is the transmission power of node $i, \alpha$ is the attenuation index, $\alpha \in[2,4][12], G_{i, j}$ is the link gain from node $i$ to node $j, N_{0}$ is AWGN power at the receiving node, $S$ is the active nodes set in the interference range, and $S_{-i}$ denotes all the active nodes except node $i$. In the deterministic formulation of the power control problem for WMNs, the AWGN power $N_{0}$ is a constant.

What a concerns selfish user is that it can utilize the channel to transmit at an optimal power level, disregarding of its impact on other transmissions. In order to optimize the QoS of WMNs, the design of utility function should consider the effect of node's transmission power on the overall performance of the network and the energy consumption, so we define the available wireless medium as

$$
A_{2}\left(p_{i}, P_{-i}\right)=\Phi-\sum_{k \in S} \xi_{k} \cdot p_{k}-N_{0}
$$

where $\Phi$ is a system constant and $\xi_{i}$ is a variable related to the source node $i$.

From a social welfare point of view, it is reasonable to charge the node some price for the consumption of energy, so we define the energy price as

$$
A_{3}\left(p_{i}, P_{-i}\right)=\mu_{i} \cdot p_{i},
$$

where $\mu_{i}$ represents the price coefficient.
We now note that the following expression is the utility function for this game:

$$
\begin{aligned}
U\left(p_{i}, P_{-i}\right)= & A_{1}\left(p_{i}, P_{-i}\right) \cdot A_{2}\left(p_{i}, P_{-i}\right)-A_{3}\left(p_{i}, P_{-i}\right) \\
= & W \cdot \log _{2}\left(1+\frac{\gamma_{i}}{\Omega}\right) \cdot\left(\Phi-\sum_{k \in S} \xi_{k} \cdot p_{k}-N_{0}\right) \\
& -\mu_{i} \cdot p_{i} .
\end{aligned}
$$

Proposition 2. An NE exits and is unique in the power control game $G=\left[N,\left\{P_{i}\right\},\left\{U_{i}(\cdot)\right\}\right]$.

Proof. The first condition of the existence of NE is satisfied obviously. We take the second-order partial derivative of utility function with respect to $p_{i}$ :

$$
\frac{\partial^{2} U\left(p_{i}, P_{-i}\right)}{\partial p_{i}^{2}}=-2 \xi_{i}<0 .
$$

So $U\left(p_{i}, P_{-i}\right)$ is a continuous and quasi-concave function in $p_{i}$. The proof of the uniqueness of $\mathrm{NE}$ of the game is straightforward according Debreu's theorem [13].

\section{System Model and Problem Formulation}

We select appropriate transmission power for network nodes using game theory and consider the communication situation in an interference range, and as long as the nodes share some common resources in WMNs, competition among them cannot be avoided, and thus a game is born accordingly. In order to enhance SINR to optimize QoS and save energy, we use Stackelberg model to model the power control problem.

Definition 3. Wireless signal strength attenuates with the propagation distance increases and the path loss can be defined as follows [12]:

$$
P_{r}(d)=\frac{P_{t} \cdot G_{t, r}}{d_{t, r}^{\alpha}},
$$

where $d_{t, r}$ is the distance from receiving node to source node, $\alpha$ is the attenuation index, and $\alpha \in[2,4] . P_{t}$ is transmission power and $G_{t, r}$ is the link gain.

To the receiving node $r$ of link $l(s, r)$, its total received signal strength is

$$
\mathrm{TE}_{r}=\sum_{i \in S_{-t}} \frac{P_{i} \cdot G_{i, r}}{d_{i, r}^{\alpha}}+\frac{P_{t} \cdot G_{t, r}}{d_{t, r}^{\alpha}}+N_{0},
$$

where $P_{t} \cdot G_{t, r} / d_{t, r}^{\alpha}$ is the signal strength at receiving node $r$ and $\sum_{i \in S_{-t}}\left(P_{i} \cdot G_{i, r} / d_{i, r}^{\alpha}\right)+N_{0}$ is the cumulative interference of the receiving node $r$, which is caused by the transmissions which are simultaneous with the transmission in link $l(s, r)$ and AWGN power at the receiving node $r$.

As for the receiving nodes in WMNs, it is difficult to obtain their interfering neighbors' detail information, 


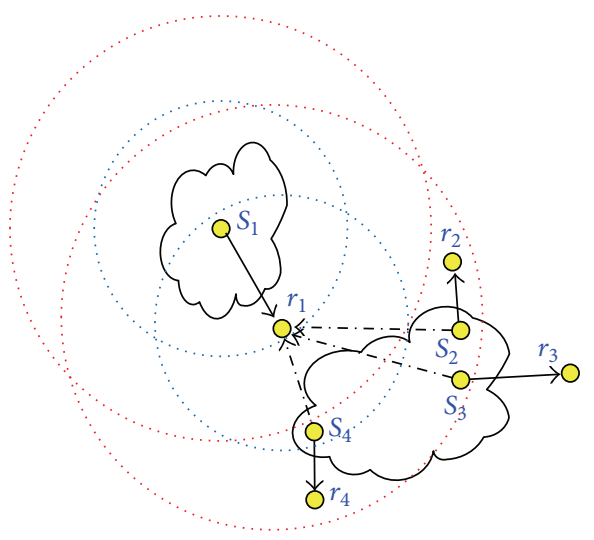

FIgURE 1: A WMNs' transmission scene.

including the transmission power and the distance to the receiving node. As the node $r_{1}$ shown in Figure 1, it may be interfered by node $s_{2}, s_{3}$, and $s_{4}$. If the system adopts some control mechanism such as RTS/CTS, the transmission $s_{4}$ in the node $r_{1}$ 's communication range will be deferred, but the transmissions of node $s_{2}$ and $s_{3}$ in the node $r_{1}$ 's interference range will impact node $r_{1}$ 's transmission all the same, and $r_{1}$ does not know them. Then, the power control problem is the power allocation between the interfering neighbors $s_{2}, s_{3}$ and the sending node $s_{1}$ of the receiving node $r_{1}$. Therefore, our goal is to design a power control scheme which can allocate transmission power properly to optimize the network throughput and save energy.

\section{Stackelberg Power Control Game (SPCG)}

5.1. Problem Formulation. Stackelberg games are also called leader-follower games. In a Stackelberg game, the players of this game are a leader and a follower. The problem assumes that the follower reacts in such a rational way that they optimize its objective function based on the leader's action. The Stackelberg model can be solved by finding the subgame perfect Nash equilibrium (SPNE).

In our power control game mode, we assume that the system adopts RTS/CTS mechanism and protocol interference model. We assume that all nodes have the same frequency and they are immobile. We assume that the selected transmission power by the proposed power control algorithm can ensure the required minimum SINR in all transmissions.

5.2. Problem Solving. According to the RTS/CTS mechanism, if the receiving node $r_{1}$ receives data, all other transmissions in the communication range of the receiving node $r_{1}$ are deferred. Thus, the interference only comes from the nodes in the region that is out of the communication range, such as $s_{2}, s_{3}$, and $s_{4}$, as shown in Figure 2, where $d_{T}$ and $d_{I}$ are the radius of the communication range and the interference range, respectively.

Because of the wireless signal attenuation characteristics, if the transmission power of the interfering nodes $s_{2}, s_{3}$, and $s_{4}$ become larger, the interference of the receiving node

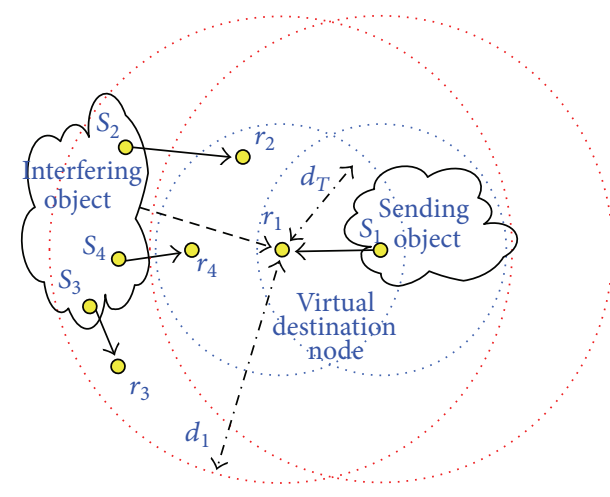

Figure 2: Wireless interference model.

$r_{1}$ that is caused by these interfering nodes will get larger, so the trend of the interference strength at the receiving node $r_{1}$ is consistent with the received signal strength at the receiving node $r_{2}, r_{3}$, and $r_{4}$. Then we define all the nodes that affect the link quality of the receiving node $r_{1}$ as an interfering object, and we define the receiving node $r_{1}$ as the interfering object's virtual destination node. Since we cannot get the interfering nodes' transmission power, we estimate the sum of the transmission power of all interfering nodes through measuring the strength of the cumulative interference at receiving node $r_{1}$. According to (2), the sum of the transmission power of all interfering nodes can be expressed by $p_{\text {inf }}=d_{\text {inf }}^{\alpha} \cdot p_{\text {inf }}^{\prime}$, where $p_{\text {inf }}^{\prime}$ is the cumulative interference of the receiving node $r_{1}$, and $d_{\text {inf }}$ is the average distance from the interfering nodes to $r_{1}$ and we define $d_{\text {inf }}=\left(d_{I}+d_{T}\right) / 2$. We regard the sum of the transmission power $p_{\text {inf }}$ of all interfering nodes as the interfering object's game strategy, which can be computed by the receiving node $r_{1}$ 's cumulative interference level $p_{\mathrm{inf}}^{\prime}$. On the other hand, we define the source node as sending object, and the transmission power level is the sending object's game strategy. So this power control process is modeled as a game between the sending object and the interfering object of the receiving node.

As the complexity restriction of power control algorithm, $A_{1}\left(p_{i}, P_{-i}\right)$ and $A_{2}\left(p_{i}, P_{-i}\right)$ are all monotone function on $p_{k}$, and $p_{k}$ is considered in $A_{2}\left(p_{i}, P_{-i}\right), k \in S_{-i}$. On the other hand, $\log _{2}\left(1+\gamma_{i} / \Omega\right)$ is a monotone function on $\gamma_{i}$, so the utility function (5) can be rewritten as

$$
\begin{aligned}
U\left(p_{i}, P_{-i}\right)= & \left(\Phi-\sum_{k \in S} \xi_{k} \cdot p_{k}-N_{0}\right) \cdot W \\
& \cdot\left(1+\frac{p_{i} \cdot G_{i, j} / d_{i, j}^{\alpha}}{\Omega \cdot\left(\sum_{k \in S_{-i}, k=1, k \neq i} G_{k, j}+N_{0}\right)}\right) \\
& -\mu_{i} \cdot p_{i} .
\end{aligned}
$$

Definition 4. The power control problem can be formulated as the Stackelberg power control game

$$
G=\left\langle O,\left\{P_{i}\right\},\left\{U_{i}(\cdot)\right\}\right\rangle,
$$


where

(1) $O=\left\{O_{1}, O_{2}\right\}$ is the set of players. $O_{1}$ is the interfering object that represents all the interfering nodes of the receiver in a transmission process; it is the leader in this game. $\mathrm{O}_{2}$ is the sending object that represents the source node; it is the follower in this game, and it reacts based on the leader's action;

(2) action set $\left\{P_{i}\right\}$; each player select power level $p_{i}$ such that $p_{i} \in P_{i}$. Player $O_{1}$ 's transmission power level is $p_{1}$, player $\mathrm{O}_{2}$ 's transmission power level is $p_{2}$;

(3) utility function $\left\{U_{i}(\cdot)\right\}$; The resulting utility level for player $O_{i}$. According the utility function in (9), considering the need of reducing the complexity of power control algorithm, we set $\xi_{k}=1$, and the utility functions of the interfering object and the sending object can be defined as follows:

$$
\begin{aligned}
U\left(p_{1}, P_{-1}\right)= & \left(\Phi-p_{1}-p_{2}-N_{0}\right) \cdot W \\
& \cdot\left(1+\frac{p_{1} \cdot G_{\mathrm{O}_{1}, r} / d_{1}^{\alpha}}{\Omega \cdot\left(G_{\mathrm{O}_{2}, r}+N_{0}\right)}\right)-\mu_{1} \cdot p_{1}, \\
U\left(p_{2}, P_{-2}\right)= & \left(\Phi-p_{1}-p_{2}-N_{0}\right) \cdot W \\
& \cdot\left(1+\frac{p_{2} \cdot G_{O_{2}, r} / d_{2}^{\alpha}}{\Omega \cdot\left(G_{\mathrm{O}_{1}, r}+N_{0}\right)}\right)-\mu_{2} \cdot p_{2},
\end{aligned}
$$

where $p_{1}$ is the transmission power of the interfering object, $p_{2}$ is the transmission power of the sending object, $d_{1}$ is the average distance from the interfering nodes to their destinations where we define $d_{1}=d_{T} / 2, d_{2}$ is the distance from the sending node to the receiving node, $G_{O_{1}, r}$ is the link gain from the interfering object $O_{1}$ to the receiving node $r$, and $G_{\mathrm{O}_{2}, r}$ is the link gain from the sending object $\mathrm{O}_{2}$ to the receiving node $r$.

Substituting $p_{1}, p_{2}$ with $p_{1}^{\prime}$, $p_{2}^{\prime}$, the Stackelberg power control game in (11) can be rewritten as follows:

$$
\begin{aligned}
U\left(p_{1}, P_{-1}\right)= & \left(\Phi-p_{1}^{\prime} \cdot d_{\mathrm{inf}}^{\alpha}-p_{2}^{\prime} \cdot d_{2}^{\alpha}-N_{0}\right) \cdot W \\
& \cdot\left(1+\frac{p_{1}^{\prime} \cdot d_{\mathrm{inf}}^{\alpha} \cdot G_{\mathrm{O}_{1}, r} / d_{1}^{\alpha}}{\Omega \cdot\left(G_{\mathrm{O}_{2}, r}+N_{0}\right)}\right)-\mu_{1} \cdot p_{1}^{\prime} \cdot d_{\mathrm{inf}}^{\alpha}, \\
U\left(p_{2}, P_{-2}\right)= & \left(\Phi-p_{1}^{\prime} \cdot d_{\mathrm{inf}}^{\alpha}-p_{2}^{\prime} \cdot d_{2}^{\alpha}-N_{0}\right) \cdot W \\
& \cdot\left(1+\frac{p_{2}^{\prime} \cdot G_{\mathrm{O}_{2}, r}}{\Omega \cdot\left(G_{\mathrm{O}_{1}, r}+N_{0}\right)}\right)-\mu_{2} \cdot p_{2}^{\prime} \cdot d_{2}^{\alpha},
\end{aligned}
$$

where $p_{1}^{\prime}=p_{1} / d_{\text {inf }}^{\alpha}$ is the cumulative interference level of the receiving node, $p_{2}^{\prime}=p_{2} / d_{2}^{\alpha}$ is the received signal strength at the receiving node, $d_{\text {inf }}$ is the average interference distance from all the interfering nodes to the receiving node, and $d_{2}$ is the distance from the sending node to the receiving node.

The SPNE can be found by solving the Stackelberg power control game model. As the power strategy of $\mathrm{O}_{2}$ depends on the power level of $O_{1}$, we define that $p_{2}^{\prime}=R\left(p_{1}^{\prime}\right)$ denotes the follower's best action function, where the subscript 1 represents the leader and 2 represents the follower. As players tend to be "selfish" and maximize their own utility, we take the first-order derivative of the follower $\mathrm{O}_{2}$ 's utility function $U\left(p_{2}, P_{-2}\right)$ with respect to its strategy $p_{2}^{\prime}$, we arrive at:

$$
\begin{aligned}
\frac{\partial U\left(p_{2}, P_{-2}\right)}{\partial p_{2}^{\prime}}= & \frac{W \cdot G_{\mathrm{O}_{2}, r}}{\Omega \cdot\left(G_{\mathrm{O}_{1}, r}+N_{0}\right)} \\
& \cdot\left(\Phi-p_{1}^{\prime} \cdot d_{\mathrm{inf}}^{\alpha}-2 p_{2}^{\prime} \cdot d_{2}^{\alpha}-N_{0}\right) \\
& -W \cdot d_{2}^{\alpha}-\mu_{2} \cdot d_{2}^{\alpha} .
\end{aligned}
$$

Define $\partial U\left(s_{2}, s_{-2}\right) / \partial p_{2}^{\prime}=0$; then we get the best action function as

$$
\begin{aligned}
p_{2}^{\prime}= & R\left(p_{1}^{\prime}\right) \\
= & \frac{1}{2 d_{2}^{\alpha}}\left(\Phi-p_{1}^{\prime} \cdot d_{\mathrm{inf}}^{\alpha}-N_{0}\right) \\
& -\frac{\Omega \cdot\left(G_{\mathrm{O}_{1}, r}+N_{0}\right) \cdot\left(W+\mu_{2}\right)}{2 W \cdot G_{\mathrm{O}_{2}, r}} .
\end{aligned}
$$

Substitute $p_{2}^{\prime}$ in $U\left(p_{1}, P_{-1}\right)$ with the $R\left(p_{1}^{\prime}\right)$ in the above formula; we get

$$
\begin{aligned}
U\left(p_{1}, P_{-1}\right)=\frac{W}{2}\left[\Phi-p_{1}^{\prime} \cdot d_{\mathrm{inf}}^{\alpha}-N_{0}\right. \\
\left.+\frac{\Omega \cdot\left(G_{\mathrm{O}_{1}, r}+N_{0}\right) \cdot\left(W+\mu_{2}\right) \cdot d_{2}^{\alpha}}{W \cdot G_{\mathrm{O}_{2}, r}}\right] \\
\\
\quad\left(1+\frac{p_{1}^{\prime} \cdot d_{\mathrm{inf}}^{\alpha} \cdot G_{\mathrm{O}_{1}, r} / d_{1}^{\alpha}}{\Omega \cdot\left(G_{\mathrm{O}_{2}, r}+N_{0}\right)}\right)-\mu_{1} \cdot p_{1}^{\prime} \cdot d_{\mathrm{inf}}^{\alpha} .
\end{aligned}
$$

We take the first-order derivative of the follower $O_{1}$ 's utility function $U\left(p_{1}, P_{-1}\right)$ with respect to $p_{1}^{\prime}$; we arrive at

$$
\begin{aligned}
& \frac{\partial U\left(p_{1}, P_{-1}\right)}{\partial p_{1}^{\prime}} \\
& =\frac{W \cdot G_{\mathrm{O}_{1}, r} \cdot d_{\mathrm{inf}}^{\alpha} / d_{1}^{\alpha}}{2 \Omega \cdot\left(G_{\mathrm{O}_{2}, r}+N_{0}\right)} \\
& \quad \times\left[\Phi-2 p_{1}^{\prime} \cdot d_{\mathrm{inf}}^{\alpha}-N_{0}+\frac{\Omega \cdot\left(G_{\mathrm{O}_{1}, r}+N_{0}\right) \cdot\left(W+\mu_{2}\right)}{W \cdot G_{\mathrm{O}_{2}, r}}\right] \\
& \quad-\frac{W}{2} \cdot d_{\mathrm{inf}}^{\alpha}-\mu_{1} \cdot d_{\mathrm{inf}}^{\alpha} .
\end{aligned}
$$


Define $\partial U\left(p_{1}, P_{-1}\right) / \partial p_{1}^{\prime}=0$; then

$$
\begin{gathered}
p_{1}=\frac{1}{2}\left[\Phi-N_{0}-\frac{\Omega \cdot\left(G_{O_{2}, r}+N_{0}\right) \cdot\left(W+2 \mu_{1}\right) \cdot d_{1}^{\alpha}}{W \cdot G_{O_{1}, r}}\right. \\
\left.\quad+\frac{\Omega \cdot\left(G_{O_{1}, r}+N_{0}\right) \cdot\left(W+\mu_{2}\right)}{W \cdot G_{O_{2}, r}}\right] .
\end{gathered}
$$

According to the best action function, we get

$$
\begin{gathered}
p_{2}=\frac{1}{4}\left[\Phi-N_{0}+\frac{\Omega \cdot\left(G_{\mathrm{O}_{2}, r}+N_{0}\right) \cdot\left(W+2 \mu_{1}\right) \cdot d_{1}^{\alpha}}{W \cdot G_{\mathrm{O}_{1}, r}}\right. \\
\left.-\frac{3 \Omega \cdot\left(G_{\mathrm{O}_{1}, r}+N_{0}\right) \cdot\left(W+\mu_{2}\right)}{W \cdot G_{\mathrm{O}_{2}, r}}\right] .
\end{gathered}
$$

So the Stackelberg equilibrium (theoretical optimal solution) of SPCG is $\left(p_{1}, p_{2}\right)$.

According to Stackelberg power control game, we know the relationship of the receiving node's cumulative interference $p_{1}^{\prime}$ and the sending node's transmission power $p_{2}$ is as follows:

$$
\begin{gathered}
p_{2}=d_{2}^{\alpha} \cdot p_{2}^{\prime}=d_{2}^{\alpha} \cdot R\left(p_{1}^{\prime}\right)=\frac{1}{2}\left(\Phi-p_{1}^{\prime} \cdot d_{\mathrm{inf}}^{\alpha}-N_{0}\right) \\
-\frac{\Omega \cdot d_{2}^{\alpha} \cdot\left(G_{\mathrm{O}_{1}, r}+N_{0}\right) \cdot\left(W+\mu_{2}\right)}{2 W \cdot G_{\mathrm{O}_{2}, r}} .
\end{gathered}
$$

\section{Stackelberg-Game-Based Power Control Algorithm}

6.1. Power Control Algorithm. One shortcoming of power control mechanisms previously based on pricing is that they do not have a convenient algorithm for implementing it in practice, and we present an efficient and straightforward power control algorithm based on game theory.

According to (19), the cumulative interference $p_{1}^{\prime}$ of the receiving node is determined in a certain network condition, and we can measure it in MAC layer, and the transmission power level can be adjusted as follows:

$$
\begin{aligned}
p_{t}= & d_{\mathrm{tra}}^{\alpha} \cdot p_{t}^{\prime}=d_{\mathrm{tra}}^{\alpha} \cdot R\left(p_{\mathrm{inf}}^{\prime}\right) \\
= & \frac{1}{2}\left(\Phi-p_{\mathrm{inf}}^{\prime} \cdot d_{\mathrm{inf}}^{\alpha}-N_{0}\right) \\
& -\frac{\Omega \cdot d_{\mathrm{tra}}^{\alpha} \cdot\left(G_{\mathrm{O}_{1}, r}+N_{0}\right) \cdot\left(W+\mu_{t}\right)}{2 W \cdot G_{\mathrm{O}_{2}, r}}
\end{aligned}
$$

where $d_{\text {tra }}$ is the distance from the sending node to the receiving node, $p_{t}^{\prime}$ is the received signal strength at the receiving node, $p_{\text {inf }}^{\prime}$ is the cumulative interference of the receiving node, $d_{\text {inf }}$ is the average distance from the interfering nodes to the receiving node, and $\mu_{t}$ represents the price coefficient. According to (2), we can optimize network performance through selecting the sending nodes' transmission power level $p_{t}$. We can see from (20) that the transmission power $p_{t}$ is a negative monotone function on cumulative interference $p_{\text {inf }}^{\prime}$, but due to the restriction of the available of wireless medium in (3), $p_{\text {inf }}^{\prime}$ will not unlimitedly increase, and $p_{t}$ will not unlimitedly decrease; they will be adjusted to achieve equilibrium and optimize their utility function.

The interfering nodes of the current receiving node may be the sending nodes in their own transmission links, and the current sending node may also cause interference to these interfering nodes, so we select the transmission power according to the cumulative interference that comes from the interfering nodes; we must also consider the impact of the current sending node's transmission power on the other sending nodes in the interference range. According to the Stackelberg game, the proposed power control algorithm can balance all transmission nodes in the interference range to share the limited network resources and improve network performance consequently. In our algorithm, the system allocates transmission power through transmitting power emendation instruction to the interfering nodes and the sending node by the receiving node. The proposed power control algorithm is as in Algorithms 1 and 2.

6.2. Time Complexity Analysis. We assumed that $n$ is the number of the active nodes in the interference range, and $m$ stands for iterative times of SGPCA.

Firstly, for the initialization that establishes interference information tables in the CIIA, in which every node broadcasts active notice message and interference notice message, and the process can be seen as setting up directed complete graph; it takes $O(2 n \cdot(n-1))$. Each node needs to register its adjacent nodes interference information; it takes $O(n \cdot(n-1))$. Each node needs to compute the distance to its adjacent nodes which takes $O(n \cdot(n-1))$. Each node calculates its own average cumulative interference which takes $O(n)$. So the time complexity of the CIIA is $O(4 n \cdot(n-1)+n)$.

Secondly, we assume all nodes are interferential, except the sending node and the receiving node, and the algorithm SGPCA needs the receiving node to transmit power emendation instruction to the interfering nodes and the sending node which takes $O(n-1)$, and all these nodes need to adjust their transmission which also takes $O(n-1)$. If the iterative times of SGPCA is $m$, the time complexity of the SGPCA is $O(2 m \cdot(n-1))$.

So, the time complexity of Stackelberg-game-based power control algorithm is

$$
\begin{aligned}
& O(2 m \cdot(n-1)+4 n \cdot(n-1)+n) \\
& \quad=O((2 m+4 n) \cdot(n-1)+n)=O\left(n^{2}\right) .
\end{aligned}
$$

As $n$ is the number of the active nodes in interference range, and it is not large, so the power control algorithm's time complexity $\mathrm{O}\left(n^{2}\right)$ is not high and it is feasible.

\section{Numeric Result and Performance Evaluation}

In this section, we present numerical results for the algorithm presented in the previous sections. We set a typical value for 


\title{
Begin \\ Input: Routing protocol; \\ Output: Interference information table;
}

(1) If a node needs to send data, it should broadcasts active notice message in two-hop range to inform other nodes, and the receiving node record the source nodes of these control messages as the active neighbor list;

(2) Each node calculates its own average cumulative interference $p_{\text {inf }}^{\prime}$ in communication process and broadcasts interference notice message including average cumulative interference $p_{\text {inf }}^{\prime}$, the active neighbor list and the transmission power level in one-hop range through routing protocol periodically;

(3) After receiving a control message from a neighbor node, each node establishes an interference information table to record the adjacent node's average cumulative interference $p_{\text {inf }}^{\prime}$, the adjacent node's active neighbor nodes and the distance $d_{\text {tra }}$ to this adjacent node which is computed through the transmission power and the received signal strength of the control message.

End

Algorithm 1: Collect interference information algorithm (CIIA).

\begin{abstract}
Begin
Input: The strategy set $P_{i}$, utility function $U_{i}(\cdot)$;

Output: Optimal transmission power set;
\end{abstract}

(1) When node needs to send data to adjacent node, it queries the cumulative interference $p_{\text {inf }}^{\prime}$ of the destination node from the interference information table firstly, and selects a proper transmission power level $p_{t}$ using (20) based on the cumulative interference of the destination and the distance to the destination. The node transmits data with the selected transmission power level $p_{t}$;

(2) Based on the received signal strength $p_{t}$ and the average cumulative interference $p_{\text {inf }}^{\prime}$, according to the Stackelberg Subgame Perfect Nash Equilibrium, the receiving node transmits power emendation instruction to the interfering nodes and the sending node;

(3) The interfering node and the sending node adjusts its transmission power to optimize its utility function based on the received power adjust instruction.

(4) Circulation steps (2) and (3), until the two game objects' transmit power achieve steady state. End

Algorithm 2: Stackelberg-game-based power control algorithm (SGPCA).

each variable to validate the efficiency of the proposed power control scheme. We set system gain $G_{O_{1}, r}=40, G_{O_{2}, r}=60$, bandwidth $W=10.0$, Gaussian white noise $N_{0}=5.0$, price coefficient $\mu_{1}=\mu_{2}=10.0$, traffic constant $\Phi=15000.0$, channel gap $\Omega=1.0$, the attenuation index $\alpha=2$, transmission distance $d_{\text {tra }}=10.0$, the average interference distance as $d_{\text {inf }}=\left(d_{I}+d_{T}\right) / 2=20$, and the average distance from the interfering nodes to their own destination $d_{\text {itod }}=$ $d_{T} / 2=6.65$. In our experiment, the transmission model is simple: we assume that there is a transmission from the sending node to its receiving node and some interfering transmission in the interference range to impact the link quality of the transmission. Convergence of the proposed power control algorithm is defined as reaching within $0.01 \%$ of the steady-state values in power update [14].

Different from machine learning research methods, we investigate the performance of the proposed power control algorithm by using analysis and comparison. In the Stackelberg game, all players are selfish and do their best to maximize their utility, and the follower reacts in such a rational way that they optimize its utility function based on the leader's action. If the leader's action does not maximize his utility, he adjusts his action to increase his utility, and the follower adjusts his action accordingly. In the proposed power control algorithm, the receiving node gets the subgame perfect Nash equilibrium point based on the system parameters. Based on the cumulative interference that comes from the interfering nodes and the received signal strength, the receiving node sends power emendation introductions to the interfering nodes and the sending node, and the nodes adjust their transmission power. At the next iteration, the receiving node senses the updated cumulative interference and the updated received signal strength, compares it to the Subgame Perfect Nash Equilibrium point, and sends power emendation introductions again. This iteration process stops only if the system achieves steady state. We first analyzed the proposed algorithm in a feasible system to verify the iteration process's convergence. Then, we used the just enough transmission power schemes [15] and the IEEE 802.11 standard as a baseline to evaluate the performance of the proposed power control 


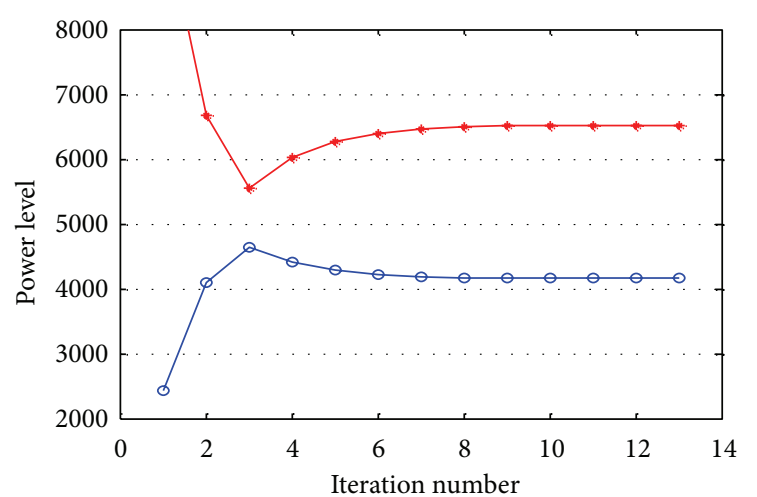

$\rightarrow \quad$ Interfering nodes' transmission power

$\rightarrow \quad$ Sending node's transmission power

FIGURE 3: The transmission power comparison.

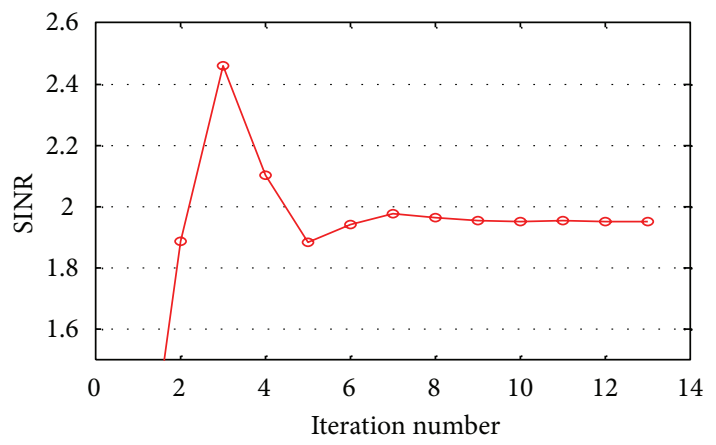

FIGURE 4: SINR of the sending node.

algorithm. We compared the proposed power control algorithm, the just enough transmission power scheme, and the IEEE 802.11 maximum transmission power scheme in three aspects, including the receiving node's SINR, energy efficient, and total utility. The just enough transmission power scheme simply modifies the IEEE 802.11 RTS/CTS mechanism, and maximum transmit power is used for request-power-tosend (RPTS)/acceptable-power-to-send (APTS) handshake between the data sender and receiver, which is used to determine the minimum transmission power that will result in a successful packet reception at the receiver for DATAACK transmission, that is, the minimum transmission power is selected based on the required minimum SINR (RMSINR) of the receiver. In our experiment, we set the RMSINR as 1.5. In the IEEE 802.11 maximal transmission power scheme, all nodes transmit data with the maximum transmit power. Also, we define the maximal transmission power ss 8000 .

7.1. Performance Evaluation of the Proposed Power Control Algorithm. According to the Stackelberg power control game, to ensure the application's QoS, the interfering node can adjust its transmission power to get the best utility, and the sending node can select transmission power based on the receiving node's cumulative interference decided by the interfering node's transmission power. The trend of the interfering node and the sending node's transmission power

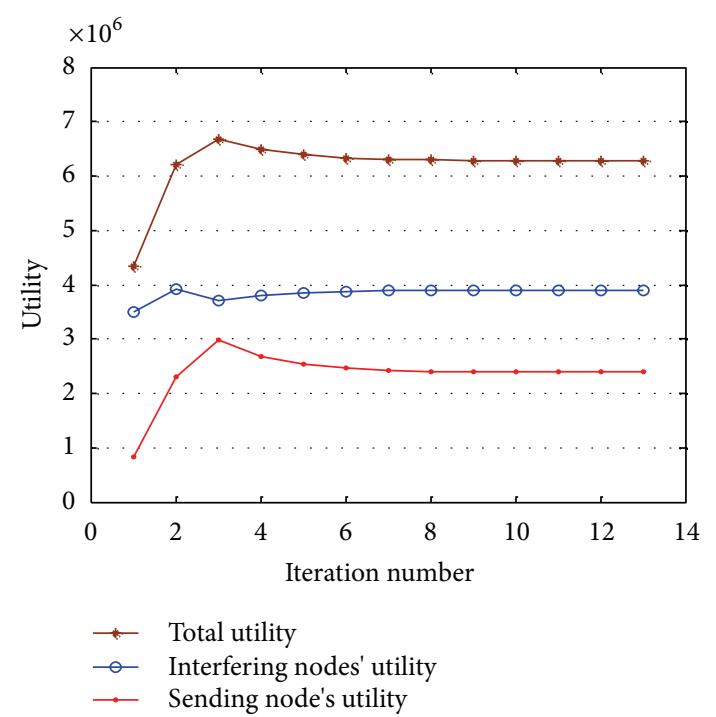

FIGURE 5: System's utility.

is shown in Figure 3. The system can converge and achieve steady state after carrying through thirteen times iteration. According to the parameter value in the aforementioned paragraph, we get the Subgame Perfect Nash Equilibrium of the power control game as $(6522,4161)$. We can see from Figure 3 that, when the system achieves steady state, the interfering nodes and the sending node's transmission power consist with the Subgame Perfect Nash Equilibrium point.

By using the same experiment model considered previously, we investigate the receiving node's SINR in iteration process, and the corresponding results are shown in Figure 4. In the third generation, the SINR is maximal, but the iteration process continues. Because at this point, the receiving node's link quality is the best, but the transmission power of the interfering node is too small. The proposed algorithm must enable all transmission nodes to share network resources evenly.

The system's utility is shown in Figure 5. We can see that the utility of the interfering node and the sending node vary with their transmission power adjustment in the iteration process. Through thirteen times iteration, each node adjusts its transmission power leading to a sequence of power vectors that converges to an equilibrium point, and there is no player that has the incentive to change its individual power. As the interfering node acts firstly and the sending node ensues, the interfering node's utility is always greater than the sending node's utility, which is called "pioneer predominance" in Stackelberg game theory.

7.2. Performance Comparison. We investigate the performance of the proposed power control algorithm in terms of SINR, energy efficiency, and users' utility. We compare the proposed algorithm with the just enough transmission power schemes and the IEEE 802.11 maximum transmission power scheme.

The comparison of the receiving node's SINR is shown in Figure 6. In the just enough transmission power schemes, 


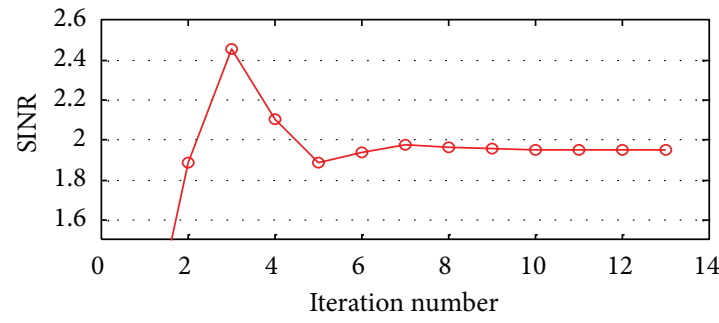

(a) The proposed power control algorithm

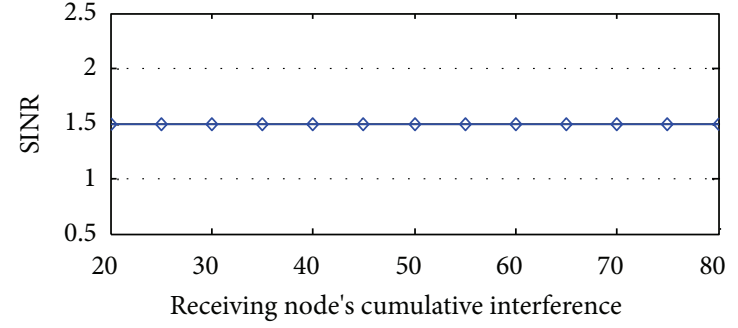

(b) The just enough transmission power scheme

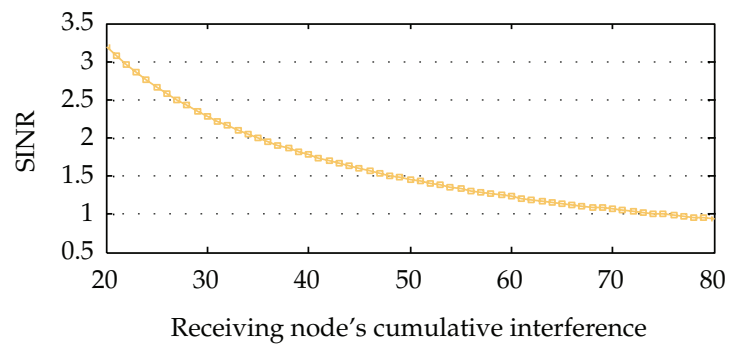

(c) IEEE 802.11 standard power control scheme

FIGURE 6: SINR comparison.

the receiving node computes the transmission power to achieve the required SINR based on its interference and informs the sending node through APTS message. The sending node transmits data with the minimum transmission power for successful transmission. The minimum required SINR is decided by the network equipment and it achieves steady, as shown in Figure 6(b). In the IEEE 802.11 maximal transmission power scheme, the transmission power is kept in the same level no matter how the wireless environment changes. The IEEE 802.11 maximum transmission power scheme desires that all nodes transmit any data with the maximum transmission power.

The SINR depraves with the increasing interference, as shown in Figure 6(c). In the proposed power control algorithm, different from the above power control schemes, the network nodes adopting the algorithm can adjust interference level through sending emendation introduction to the interfering nodes, and the power control algorithm is acclimated to new environment. The system can achieve steady state after limited number of iteration. Compared with the just enough transmission power schemes and the IEEE 802.11 standard transmission power scheme, the proposed algorithm can enable nodes to get sufficient SINR in complicated network conditions.

We define the energy efficiency as the ratio of the total utility to the sum of the transmission power of the interfering nodes and the sending node. Figure 7 shows the comparison of the three power control schemes' energy efficiency. We can see that the proposed power control algorithm's energy efficiency is stable, whereas the energy efficiency of the just enough transmission power scheme and the IEEE 802.11 maximum transmission power scheme decline rapidly with the receiving node's cumulative interference increases.

The utility comparison of the three power control schemes is demonstrated in Figure 8. In the mass, the total

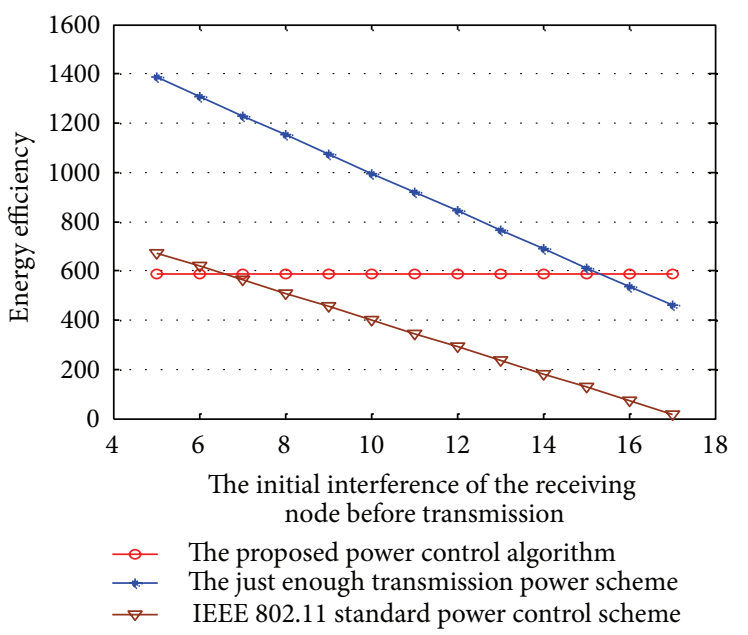

FIGURE 7: Energy efficiency comparison.

utilities of the nodes adopting the IEEE 802.11 standard control scheme are less than those of the nodes adopting the proposed power control algorithm and the just enough transmission power scheme, and the total utilities decrease with the receiving nodes' cumulative interference increases. If the receiving nodes' interference level is low, the total utilities of the nodes adopting the just enough transmission power schemes are higher than the proposed power control algorithm. But if the interference level becomes greater, the total utilities will become very small, and the just enough transmission power schemes cannot offer adequate performance to ensure QoS.

\section{Conclusion}

In this paper, we investigated the problem of power control and proposed an efficient power control algorithm for 


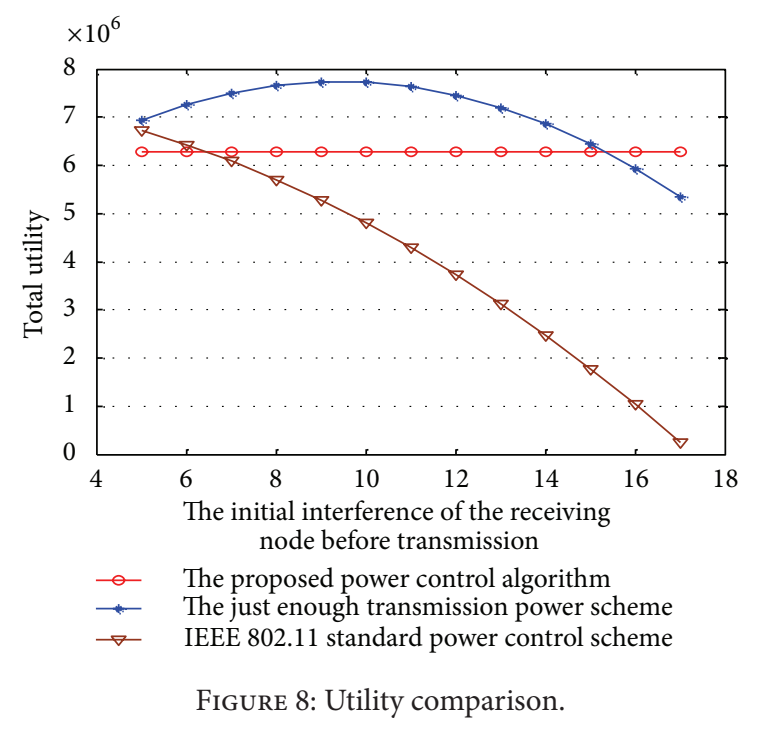

WMNs based on game theory. We modeled the power control problem to be a noncooperative Stackelberg game by observing the distinctive features of WMNs that each user makes its own decision selfishly and devised a utility function which comprehensively considers both of throughput and energy efficiency. Through the technique of SPNE in such Stackelberg game, we deduced the relationship between the sending node's transmission power and the receiving node's cumulative interference. The deduced equilibrium point indicates that the receiving node transmits power emendation instruction to the interfering nodes and the sending node to adjust their transmission power. We also presented the numerical experiment; the results show that all of the users obtain the sufficient SINR and achieve energy efficiency, and the proposed algorithm can balance nodes to share the limited network resources and maximize total utility; thus it is efficient and effective in solving the power control problem.

\section{Acknowledgments}

The work was supported by the National Program on Key Basic Research Project (973 Program) of China (Grants nos. 2010CB334710 and 2012Cb315803), National Science Foundation of China (Grant no. 61272400), NCET, R\&D Foundation of Chongqing (Grant no. Kjzh10206), and Open Project of Key Lab of Information Network Security of Administration of Public Security (Grant no. C11609).

\section{References}

[1] A. B. MacKenzie and S. B. Wicker, "Game theory and the design of self-configuring, adaptive wireless networks," IEEE Communications Magazine, vol. 39, no. 11, pp. 126-131, 2001.

[2] A. Muqattash and M. Krunz, "POWMAC: a single-channel power-control protocol for throughput enhancement in wireless ad hoc networks," IEEE Journal on Selected Areas in Communications, vol. 23, no. 5, pp. 1067-1084, 2005.

[3] J. Huang, R. A. Berry, and M. L. Honig, "Distributed interference compensation for wireless networks," IEEE Journal on
Selected Areas in Communications, vol. 24, no. 5, pp. 1074-1084, 2006.

[4] F. O. Aron, T. O. Olwal, A. Kurien et al., "A distributed topology control algorithm to conserve energy in heterogeneous wireless Mesh networks," Proceedings of World Academy of Science, Engineering and Technology, vol. 30, pp. 530-536, 2008.

[5] C. K. Tan, M. L. Sim, and T. C. Chuah, "Game theoretic approach for channel assignment and power control with nointernal-regret learning in wireless ad hoc networks," IET Communications, vol. 2, no. 9, pp. 1159-1169, 2008.

[6] C. K. Tan, M. L. Sim, and T. C. Chuah, "Fair power control for wireless ad hoc networks using game theory with pricing scheme," IET Communications, vol. 4, no. 3, pp. 322-333, 2010.

[7] E. A. Panaousis, C. Politis, and G. C. Polyzos, "Maximizing network throughput," IEEE Vehicular Technology Magazine, vol. 4, no. 3, pp. 33-39, 2009.

[8] F. Meshkati, H. V. Poor, and S. C. Schwartz, "Energy-efficient resource allocation in wireless networks," IEEE Signal Processing Magazine, vol. 24, no. 3, pp. 58-68, 2007.

[9] F. Meshkati, M. Chiang, H. V. Poor, and S. C. Schwartz, "A game-theoretic approach to energy-efficient power control in multicarrier CDMA Systems," IEEE Journal on Selected Areas in Communications, vol. 24, no. 6, pp. 1115-1129, 2006.

[10] V. Nagarajan and P. Dananjayan, "A novel game theoretic approach for energy efficient modulation in MC-DS-CDMA," in Proceedings of the International Conference on Future Computer and Communication (ICFCC'09), pp. 112-116, Kuala Lumpur, Malaysia, April 2009.

[11] X. M. Zhang, Q. Liu, S. F. Dai, and Y. Z. Liu, "Traffic loadbased interference-aware routing protocol for mobile ad hoc networks," Journal of Software, vol. 20, no. 10, pp. 2721-2728, 2009.

[12] S. Toumpis and A. J. Goldsmith, "Capacity regions for wireless ad hoc networks," IEEE Transactions on Wireless Communications, vol. 2, no. 4, pp. 736-748, 2003.

[13] G. Debreu, "A social equilibrium existence theorem," Proceedings of the National Academy of Sciences of the United States of America, vol. 38, pp. 886-893, 1952.

[14] S. Koskie and Z. Gajic, "A Nash game algorithm for SIR-based power control in 3G wireless CDMA networks," IEEE/ACM Transactions on Networking, vol. 13, no. 5, pp. 1017-1026, 2005.

[15] J. P. Monks, V. Bharghavan, and W. W. Hwu, "A power controlled multiple access protocol for wireless packet networks," in Proceedings of the 20th Annual Joint Conference on the IEEE Computer and Communications Societies (IEEE INFOCOM'01), vol. 1, pp. 219-228, Anchorage, Alaska, USA, April 2001. 


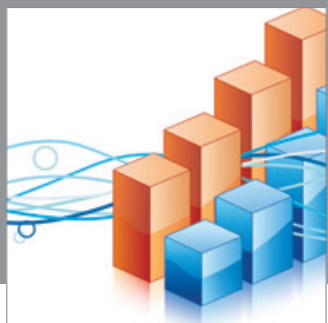

Advances in

Operations Research

mansans

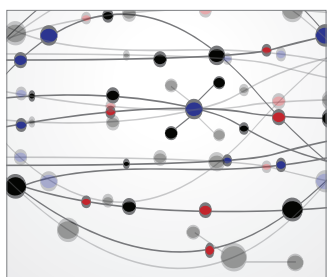

The Scientific World Journal
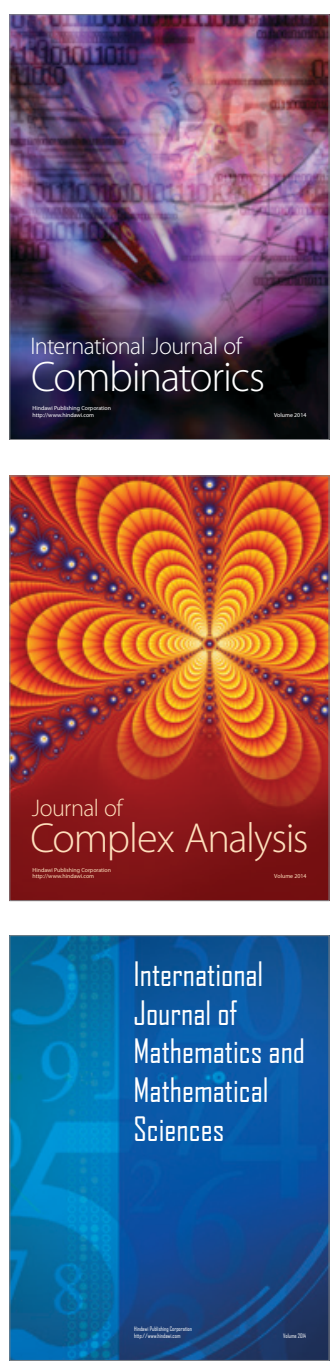
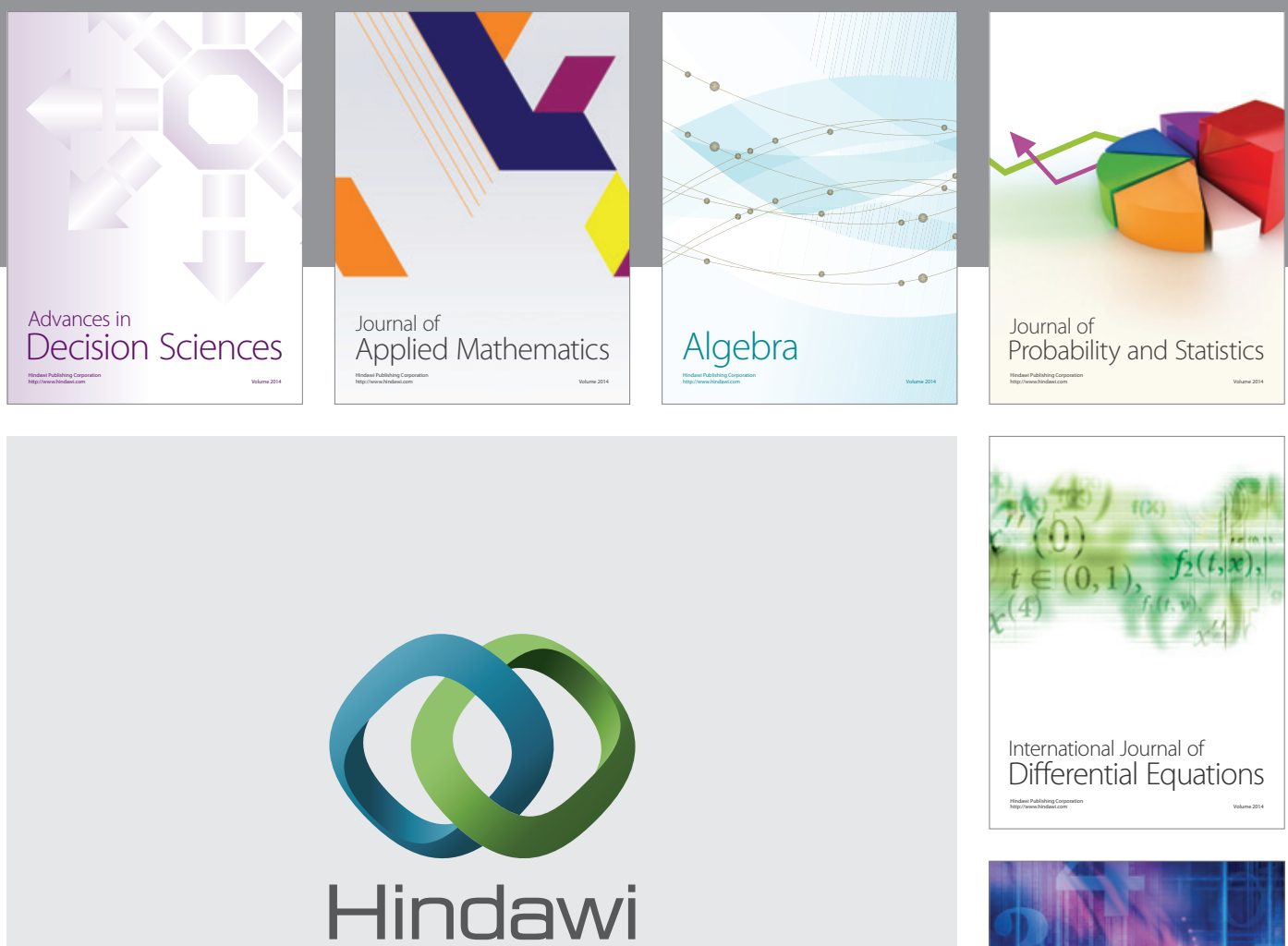

Submit your manuscripts at http://www.hindawi.com
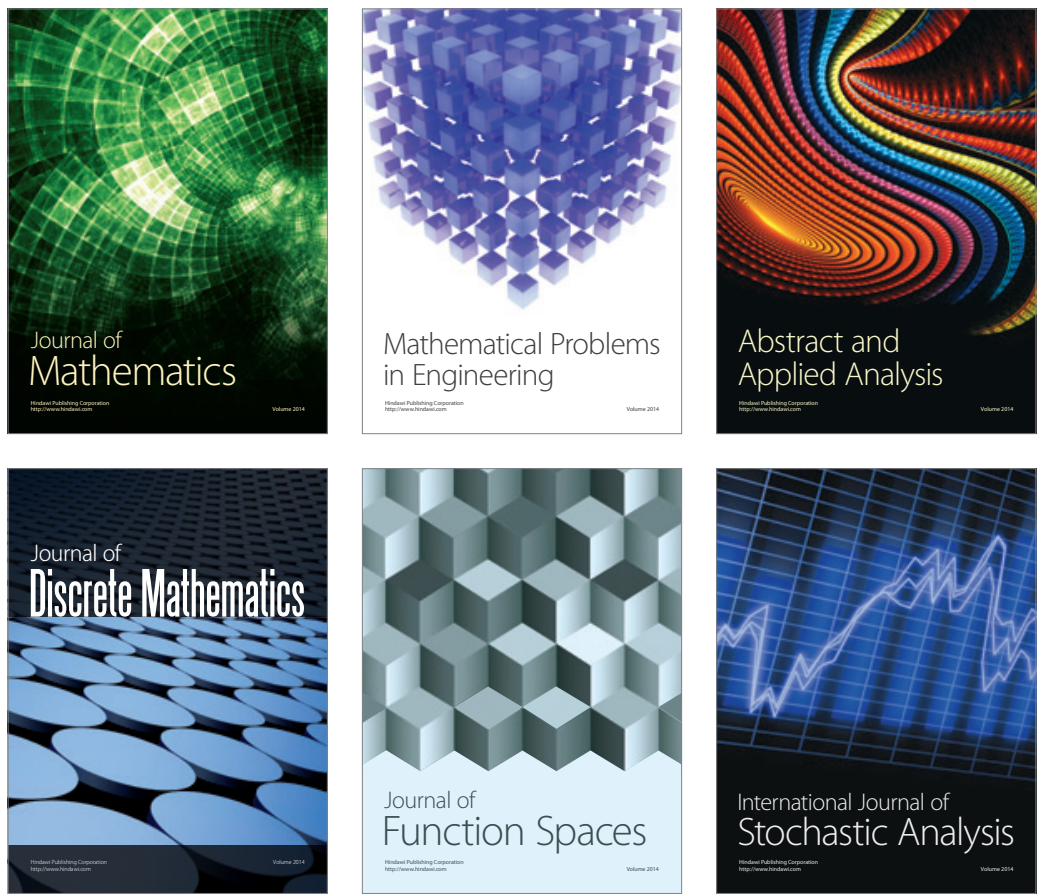

Journal of

Function Spaces

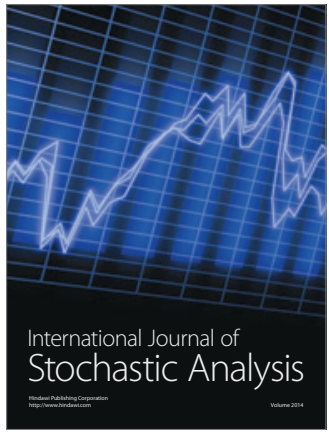

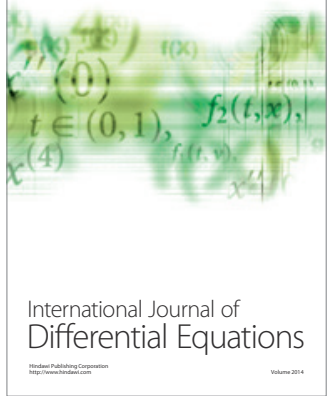
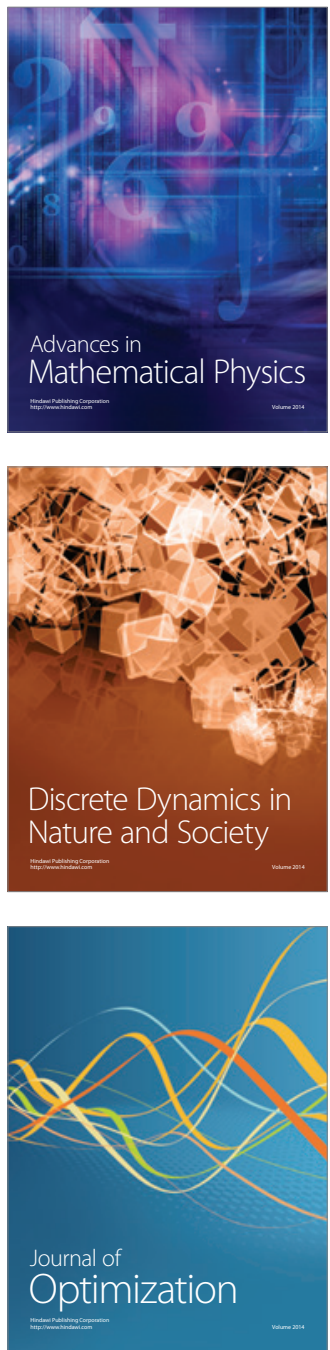